Research Article

\title{
Human Epididymis Protein 4 Levels in Neonates with Respiratory Disorder
}

\author{
Piotr Surmiak (D), Martyna Szymkowiak (D), and Małgorzata Baumert (D) \\ Department of Neonatology, Medical University of Silesia in Katowice, Faculty of Medical Sciences, Katowice 40-055, Poland \\ Correspondence should be addressed to Piotr Surmiak; piotr.surmiak@sum.edu.pl
}

Received 19 October 2019; Accepted 16 March 2020; Published 2 April 2020

Academic Editor: Maurizio Battaglia Parodi

Copyright ( 2020 Piotr Surmiak et al. This is an open access article distributed under the Creative Commons Attribution License, which permits unrestricted use, distribution, and reproduction in any medium, provided the original work is properly cited.

\begin{abstract}
Introduction. Transient tachypnea of the newborn (TTN) is one of the most common causes of respiratory distress in the full-term neonates. The diagnosis of TTN in early postnatal period remains problematic for clinicians, and unfortunately, there exist no reliable diagnostic tests for TTN. The elevated human epididymis protein 4 (HE4) levels were observed in the cases of severe bronchitis, pneumonia, and inflammatory processes. However, little is known about the fluctuation of this biomarker concentrations in respiratory disorders in neonatal period. The authors investigated the HE4 levels found in the umbilical cord blood and venous blood samples of the newborns with respiratory disorder (TTN) and blood samples of their mothers. Materials and Methods. The investigated neonates were divided into two groups: 23 neonates with the respiratory insufficiency (transient tachypnea of the newborn, TTN) as the study group and 28 newborns of healthy mothers constituted the control group (CG). The C-reactive protein (CRP) and procalcitonin (PCT) as well as HE4 levels were determined in umbilical cord blood and venous blood for all the examined neonates and their mothers. Results. There were no differences found in the HE4 levels determined for the mothers' blood samples and umbilical cord blood samples in all investigated groups. In comparison with healthy children, the elevated HE4 levels were observed in neonates with TTN. Significant positive correlation between HE4 and CRP as well as PCT levels was observed in all investigated neonates. The receiver operating characteristic (ROC) curve analysis demonstrated the cut-off value for the serum HE4 in the researched neonates at the level of $318.5 \mathrm{pmol} / \mathrm{L}$, yielding the sensitivity of $73.9 \%$ and specificity of $66.7 \%$ for the early diagnosis of TTN. Conclusions. Serum HE4 could be considered as a candidate biomarker for the early diagnosis of pulmonary dysfunction in the newborns.
\end{abstract}

\section{Introduction}

Transient tachypnea of the newborn (TTN) is one of the most common causes of respiratory distress in neonatal period, affecting $0.5 \%$ to approximately $5 \%$ of all late preterm and term neonates in the immediate postdelivery period [1-3]. TTN called wet lungs is a common self-limited disease of term newborns that results from delayed lung fluid clearance and sometimes affects babies during the first hours of life $[4,5]$. The diagnosis of TTN in early postnatal period remains problematic for clinicians. The most typical presenting symptoms, tachypnea and respiratory distress and the need for supplemental oxygen, are common among most neonatal respiratory disorders, and unfortunately, there exist no reliable diagnostic tests for TTN.
Even though there is broad interest in the biomarker research, the scientific investigation has yielded only a few new indicators (particularly cancer biomarkers) that seem valuable for the clinical use in recent years. The search for the new markers that could help to detect a specific disease is driven by the hope for the early disease detection and immediate treatment implementation to achieve the best possible results in the disease treatment. For that reason, the markers should be highly specific and sensitive.

The serum human epididymis protein 4 (HE4) is one of the biomarkers recently included in the pipeline of clinical testing [6]. HE4 (also known as the WAP four-disulfide core domain protein 2, WFDC2) is a N-glycosylated protein, which is encoded by the gene located on the chromosome 20q12-13.1. The protein was first identified as a transcript 
TABLE 1: Demographic and perinatal characteristics of the investigated neonate groups. Results presented as a median and [95\% confidence interval for median] or percentile.

\begin{tabular}{lccc}
\hline Investigated group & Neonates with TTN $(n=23)$ & Controls $(n=28)$ & $p$ value \\
\hline Mothers' age (years) & $29[26-33]$ & $30[26-31]$ & 0.34 \\
Gestational age (weeks) & $38[37-39]$ & $39[38-40]$ & $12 ; 42.9 \%$ \\
Gender: female (no.; \%) & $9 ; 39.1 \%$ & $16 ; 57.1 \%$ & 0.62 \\
Gender: male (no.; \%) & $14 ; 60.9 \%$ & $3145[3040-3320]$ & $0.21^{*}$ \\
Birth weight (g) & $3240[3010-3510]$ & $0.38^{*}$ \\
Apgar score (no.; \%) & & $5 ; 17.9 \%$ & 0.46 \\
(i) $0-3$ points & 0 & $23 ; 82.1 \%$ \\
(ii) 4 -7 points & $5 ; 21.7 \%$ & & $0.33^{*}$ \\
(iii) 8 -10 points & $18 ; 78.3 \%$ & & \\
\hline
\end{tabular}

*The $p$ value from the Mann-Whitney $U$ test or chi-squared test.

exclusively expressed in the epididymis and concerned with the maturation of sperm [7]. HE4 is a good example of a candidate biomarker originating from the gene expression analysis that was later translated into the clinical practice.

In recent years, the number of articles on the diagnostic utility of HE4 levels (e.g., in the ovarian cancer diagnostics) has considerably increased [8]. Some authors reported the elevated HE4 levels in the cases of other diseases (such as renal fibrosis and chronic kidney disease, cystic fibrosis, lung cancer, and the degree of the pulmonary dysfunction) [9-13].

Based on above, the authors of this study decided to investigate the HE4 levels in the umbilical cord blood and venous blood of the neonates with respiratory disorder (transient tachypnea of the newborn) and healthy newborns.

\section{Material and Methods}

2.1. Study Population. The researched sample came from a single-center prospective cohort study, approved by the Bioethics Committee of the Medical University of Silesia in Katowice (no. KNW/022/KB1/151/I/16/17). All the parents provided a signed informed consent before recruitment into the research. The neonatal care and monitoring were performed as part of the standard hospital protocol. Among the 1132 children born in the Department of Neonatology (Medical University of Silesia in Katowice, Poland) between May 1, 2017, and May 31, 2018, 51 full-term newborn babies (37-41 hbd) were enrolled in this study.

The investigated neonates were divided into two groups: 23 neonates with the respiratory insufficiency (transient tachypnea of the newborn, TTN) as the study group and 28 newborn babies of healthy mothers constituted the control group (CG). All neonates suffered TTN immediately after delivery, and the diagnosis was based on the clinical symptoms and chest X-ray.

The neonates that were small for the gestational age; the ones with congenital abnormalities or chromosomal anomalies, metabolic disorders, and congenital infections; and the ones from multiple pregnancies were excluded from the study. Newborns from mothers who suffered from diabetes mellitus and asthma were also excluded from this investigation. The information on the subject demographics, clinical history, and relevant comorbidities was collected upon the enrollment.

The demographic and perinatal characteristics of the study population are presented in Table 1.

2.2. Research Methods. During the delivery, $2 \mathrm{~mL}$ of the maternal venous blood and $1 \mathrm{~mL}$ of the umbilical artery blood were collected. The neonatal blood samples $(0.5 \mathrm{~mL})$ were collected from the peripheral vessels during a routine blood sampling procedure 24 hours after the birth.

The C-reactive protein (CRP) and procalcitonin (PCT) concentrations were determined for all the examined neonates immediately after the delivery. The C-reactive protein values were measured with the immunoturbidimetry method (Beckman Coulter AU analyzer). The procalcitonin levels were determined with the electrochemiluminescence method (ECLIA) (Cobas E 601 analyzer). The blood samples for the HE4 measurements were collected, centrifuged for 10 minutes (2500 rotations $/ \mathrm{min}$ ), and stored at $-80^{\circ} \mathrm{C}$ until the full analysis was performed. The serum HE4 levels were determined with the sandwich-enzyme immunoassay (ELISA) (BioVendor kit; Brno, Czech Republic). The ELISA allows for quantitative measurements of the HE4 levels in the serum. It has a detection limit of $0.15 \mathrm{pmol} / \mathrm{L}$.

2.3. Statistical Analysis. The normal distribution of the data was verified with the Shapiro-Wilk test. The continuous variables among the groups were compared with the Kruskal-Wallis test or the Mann-Whitney $U$ test due to the nonparametric nature of the data. The categorical variables were compared with the chi-squared test. The quantitative variables are presented as a median with $95 \%$ confidence intervals for the median $(95 \% \mathrm{CI})$, whereas the qualitative variables are given as percentage values. The Spearman correlation coefficients were calculated between the laboratory HE4 measurements and clinical variables. The HE4 levels are expressed as a median and 95\% confidence intervals for the median $(95 \% \mathrm{CI})$ due to the nonparametric nature of the data.

The predictive performance was assessed by means of the area under the receiver operating characteristic (ROC) curve. The ROC curves and area under the curves (AUC) with 
TABLE 2: Serum levels of human epididymis protein 4 (HE4), C-reactive protein (CRP), and procalcitonin (PCT) in the mothers' blood samples, umbilical cord blood samples, and neonatal blood samples collected 24 hours after the delivery. Results presented as a median and $[95 \%$ confidence interval for median].

\begin{tabular}{lccc}
\hline Investigated group & TTN $(n=23)$ & Controls $(n=28)$ & $p$ value \\
\hline Mothers' HE4 levels $(\mathrm{pmol} / \mathrm{L})$ & $52.0[42.8-74.8]$ & $48.7[43.8-63.1]$ & 0.65 \\
Umbilical HE4 levels $(\mathrm{pmol} / \mathrm{L})$ & $235.8[193.9-271.1]$ & $204.5[186.2-238.5]$ & 0.14 \\
Neonatal HE4 levels $(\mathrm{pmol} / \mathrm{L})$ & $383.1[312.8-511.7]$ & $305.4[284.3-370.1]$ & $<0.001^{*}$ \\
$p$ value & $<0.0001^{*}$ & $5.2[3.4-6.0]$ & 0.003 \\
Mothers' CRP levels $(\mathrm{mg} / \mathrm{L})$ & $4.6[3.2-6.3]$ & $0.3[0.1-0.3]$ & 0.52 \\
Mothers' PCT levels $(\mathrm{ng} / \mathrm{mL})$ & $0.4[0.1-0.4]$ & $1.3[0.7-2.1]$ & 0.62 \\
Neonatal CRP levels $(\mathrm{mg} / \mathrm{L})$ & $1.8[0.9-3.1]$ & $0.7[0.5-1.4]$ \\
Neonatal PCT levels $(\mathrm{ng} / \mathrm{mL})$ & $0.6[0.4-1.2]$ & 0.11 \\
\hline
\end{tabular}

${ }^{*}$ The $p$ value from the Mann-Whitney $U$ test and Kruskal-Wallis test.

Youden index were used to assess the diagnostic accuracy of the serum HE4 for predicting transient tachypnea of the newborn.

The statistical analysis was based on the standard procedures available in STATISTICA 13.3 (Statsoft Polska Inc.) and MedCalc Software 12.7.4. The statistical inferences were based on the level of significance $(p<0.05)$.

\section{Results}

The mothers and newborns from the study group (TTN) were compared with the controls. The demographic and perinatal characteristics were taken into consideration. There were no significant differences in birth weight, gender, and Apgar score between the group of neonates with TTN compared with controls (Table 1).

We did not observe any differences in the HE4 concentrations in the mothers' blood samples $(p=0.65)$ or in the umbilical cord blood samples $(p=0.14)$ between the TTN and control groups. The significantly higher HE4 concentration values in the neonatal blood (collected 24 hours after the delivery) were observed in comparison with the level determined for the umbilical cord blood in both investigated groups $(p<0.0001)$.

When compared with controls, 23 neonates with the respiratory insufficiency (TTN) demonstrated significantly higher HE4 levels $(p=0.003)$. The respective results are presented in Table 2 and Figure 1.

There were no differences among the investigated groups when it comes to the CRP and PCT levels in the mothers' and neonatal venous blood samples collected 24 hours after delivery in the TTN and control groups (Table 2).

When taking into account the neonates' gender, there were no differences in the HE4 concentrations in either the umbilical cord blood samples or the neonatal venous blood samples collected 24 hours after the delivery (Table 3 ).

First, the HE4, CRP, and PCT levels were correlated in all the groups. A significant positive correlation was detected between the HE4 and CRP values $(r=0.57 ; p<0.001)$ in all neonates. The results are presented in Figure 2. Additionally, significant correlation was found between the HE4 and pro-

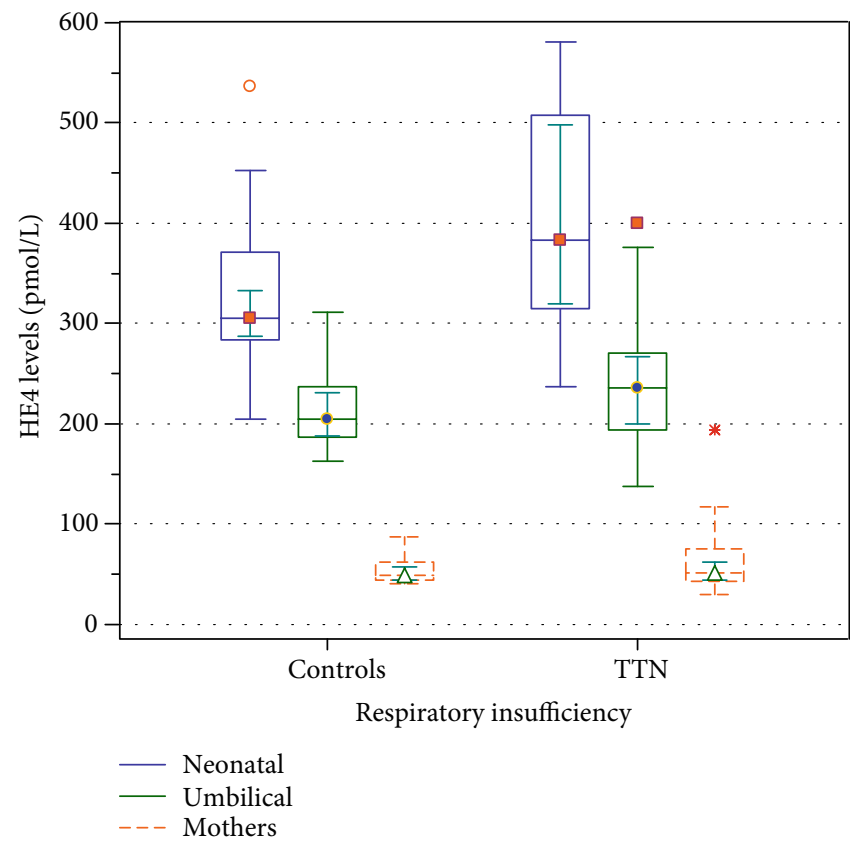

FIgURe 1: Serum HE4 levels in mothers' blood samples, umbilical cord blood samples, and neonatal blood samples collected 24 hours after the delivery in the neonates with respiratory insufficiency (transient tachypnea of the newborn, TTN) and controls. Results presented as a median as well as $95 \%$ confidence intervals for median and extreme values.

calcitonin concentrations $(r=0.41 ; p=0.02)$ in the studied newborns, shown in Figure 3.

We did not observe any correlation between CRP, PCT, and HE4 levels in mothers' blood samples.

To determine the performance of HE4 as a prognostic biomarker, the AUC analysis with Youden index was performed. The cut-off value with the Youden index for the serum HE4 in the neonates 24 hours after the delivery was $318.5 \mathrm{pmol} / \mathrm{L}$, yielding the sensitivity of $73.9 \%$ (95\% CI $51.6-89.8 \%)$ and specificity of $66.7 \%$ (95\% CI 46.0-83.5\%) for the early diagnosis of TTN (area under the ROC curve, AUC $=0.75$ [95\% CI 0.60-0.86]; $Z$ statistic 3.5; $p<0.0001$; Youden index $J=0.41$ ). The results are given in Figure 4 . 
TABle 3: Serum HE4 levels from the umbilical cord blood samples and neonatal blood samples collected 24 hours after the delivery, depending on the neonates' gender. Results presented as a median and [ $95 \%$ confidence interval for median]; the $p$ value from the MannWhitney $U$ test.

\begin{tabular}{lccc}
\hline Investigated group & Female neonates $(n=21)$ & Male neonates $(n=30)$ & $p$ value \\
\hline Neonatal HE4 levels $(\mathrm{pmol} / \mathrm{L})$ & $350.8[285.3-450.4]$ & $322.0[250.1-406.8]$ & 0.48 \\
Umbilical HE4 levels $(\mathrm{pmol} / \mathrm{L})$ & $257.9[221.2-286.3]$ & $227.2[195.7-271.3]$ & 0.33 \\
\hline
\end{tabular}

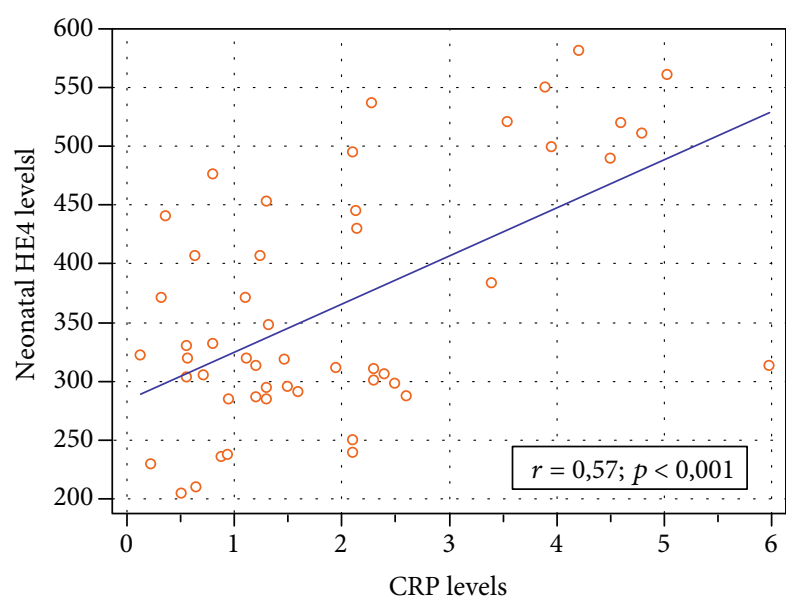

FIgURE 2: Correlation chart of the serum HE4 (pmol/L) and CRP $(\mathrm{mg} / \mathrm{L})$ levels in the neonates' blood collected 24 hours after the delivery.

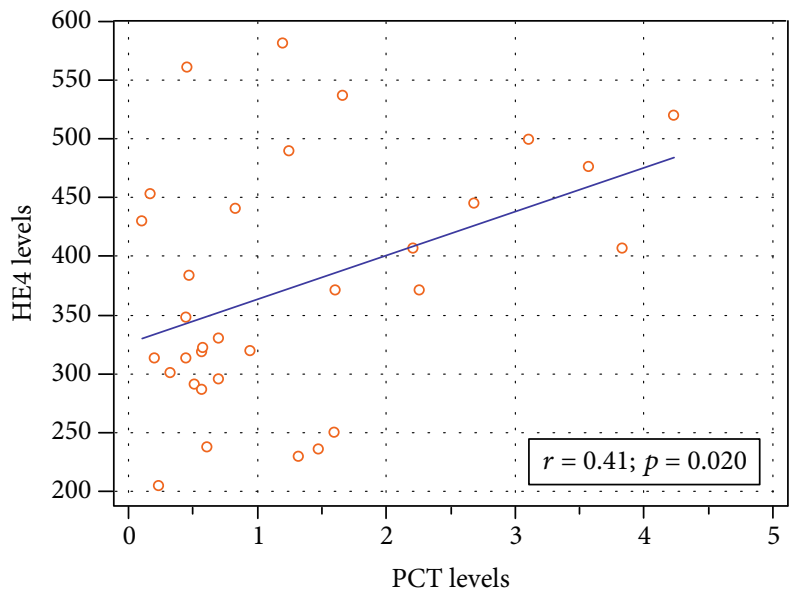

FIGURE 3: Correlation chart of the serum HE4 (pmol/L) and PCT $(\mathrm{ng} / \mathrm{mL})$ levels in the neonates' blood collected 24 hours after the delivery.

\section{Discussion}

Cancer biomarkers have become an important tool in the treatment of cancer patients, particularly in the monitoring of patients during and after the treatment $[14,15]$. Recently, the serum HE4 has been identified as a diagnostic and prognostic biomarker for lung cancer, lung malignancies, ovarian carcinoma, and endometrial carcinomas [16, 17]. The HE4 levels may also be elevated in certain noncancer diseases,

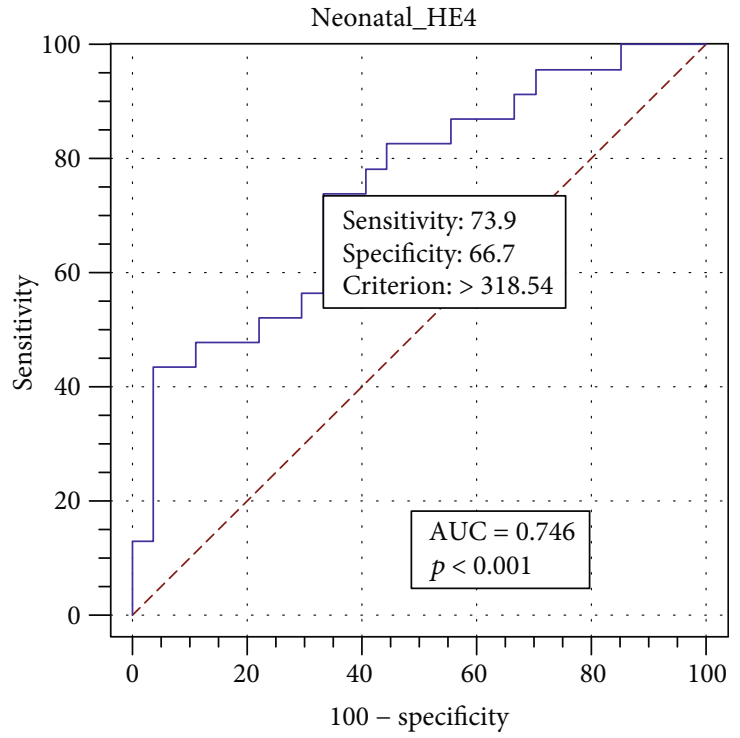

FIGURE 4: Receiver operating characteristic (ROC) curve used to determine the serum HE4 cut-off value with the Youden index for the TTN detection. The area under the curve (AUC) value for the serum HE4 was 0.75 (95\% CI 0.60-0.86); $Z$ statistic 3.50; +LR 2.22 (95\% CI 1.20-4.00); -LR 0.39 (95\% CI $0.20-0.79$ ) and $p<0.001$; Youden index $J=0.41$.

such as the chronic kidney disease or heart failure severity $[9,18]$. Significant increases in the expression and localization of the WFDC2 were observed in patients with cystic fibrosis. Additionally, the abnormal HE4 concentrations were found in the cases of severe bronchitis, asthma, pneumonia, and inflammatory processes $[10,19]$. Nonetheless, little is known about the fluctuation of the cancer marker concentrations in a healthy reference population, especially in children and neonates $[20,21]$.

As there are no reference intervals, the authors of this article decided to study the full-term neonates with respiratory disorder (TTN) so as to evaluate the HE4 concentration in the umbilical cord blood and venous blood ( 24 hours after the delivery). In the study, the mean HE4 values determined for the 51 examined newborn babies were $355.2 \mathrm{pmol} / \mathrm{L}$ with $95 \%$ confidence intervals for the median of $301.1 \mathrm{pmol} / \mathrm{L}$ to $369.9 \mathrm{pmol} / \mathrm{L}$. There was no significant correlation between HE4 levels and neonates' gender. In the adult population, the research in this field is not conclusive. Lamy et al. confirmed the results obtained in this study, but Hertlein et al. suggested that the serum HE4 levels differed between genders $[22,23]$.

The HE4 concentrations during the delivery were also studied. The mean HE4 level in the mothers was $49.7 \mathrm{pmol} / \mathrm{L}$ 
with $95 \%$ confidence intervals for the median of $45.6 \mathrm{pmol} / \mathrm{L}$ to $57.9 \mathrm{pmol} / \mathrm{L}$. Similar results were presented by other authors $[24,25]$.

The importance of this study lies in the fact that the HE4 levels were measured for the first time in the umbilical cord blood samples. The significantly higher umbilical HE4 levels in the entire study group were observed in comparison with the mothers' blood levels. It can be assumed that the majority of HE4 is of fetal origin in the cord blood, as this marker is not produced by the placenta [26].

An important finding was the increase in the neonatal HE4 levels determined 24 hours after the delivery. Under physiological conditions, the highest expression of the WFDC2 gene occurs in the lung tissue and endocrine glands $[12,13,26]$. During the delivery, there occur hormonal changes which probably stimulate the expression of this gene in the endocrine glands, which translates into the elevation of the HE4 concentrations in the umbilical cord blood. The first breath and further respiration of the child stimulate the gene expression in the lung tissue, resulting in the higher HE4 levels in the neonate venous blood.

The previous research demonstrates that some oncofetal antigens (HE4, alpha-fetoprotein (AFP), and carcinoembryonic antigen (CEA)) exhibit the expected pattern of expression with high concentrations at birth, followed by rapid decreases to significantly lower concentrations. This pattern is likely explained with the fact that many of the analytes are expressed during the fetal development and/or play an important role in the early postnatal development $[20,27]$. It is also possible that these analytes are found in higher concentrations in the serum of neonates due to the immature hepatic function. In other words, if the liver processes these proteins, the high serum concentrations observed here may be the result of the inefficiency in immature neonatal livers [20].

Perhaps, HE4 plays an important role in the physiological fetal growth conditions, although it is recognized as a cancer biomarker. It may be similar to the alpha-fetoprotein, whose concentration is determined in the physiological pregnancy. However, the most characteristic increase is observed in malignant tumors, e.g., liver, testes, and ovaries [28].

In this study, higher levels of HE4 in the neonates with respiratory disorder were observed. It is well known that an inflammatory process may play an important role in the respiratory disorders (including TTN) [29]. Thanks to the previously published articles, it is acknowledged that the WDFC protein (to which HE4 belongs) is overexpressed in the inflammatory process. Therefore, it should be assumed that the higher HE4 concentrations observed in the newborn babies with TTN may be the result of the inflammatory process within the lungs. The obtained observations were confirmed with the ROC analysis results, which demonstrated not very high sensitivity and specificity for the early diagnosis of pulmonary dysfunction in the neonates due to the determination of the serum HE4 levels. We believe that serum HE4 levels could be considered as a candidate for early biochemical marker of respiratory insufficiency in newborns.

\section{Conclusion}

The obtained results may lead to a conclusion that the serum HE4 could be considered as a candidate biomarker for the early diagnosis of pulmonary dysfunction in newborns. This study is the first one to focus on the HE4 concentration analysis in the neonatal population; thus, its results should be confirmed with further studies in this field.

\section{Data Availability}

The data used to support the findings of this study are available from the corresponding author upon request.

\section{Conflicts of Interest}

The authors declare that there is no conflict of interest regarding the publication of this paper.

\section{Acknowledgments}

This study was supported by the Medical University of Silesia in Katowice, Poland (statutory work of Dept. of Neonatology, number KNW-1-134/N/7/K).

\section{References}

[1] S. Heinonen, L. Süvari, M. Gissler, O. Pitkänen, S. Andersson, and O. Helve, "Transient tachypnea of the newborn is associated with an increased risk of hospitalization due to respiratory syncytial virus bronchiolitis," The Pediatric Infectious Disease Journal, vol. 38, no. 4, pp. 419-421, 2019.

[2] V. Zanardo, A. K. Simbi, M. Franzoi, G. Soldà, A. Salvadori, and D. Trevisanuto, "Neonatal respiratory morbidity risk and mode of delivery at term: influence of timing of elective caesarean delivery," Acta Paediatrica, vol. 93, no. 5, pp. 643647, 2004.

[3] L. Jain, "Respiratory morbidity in late-preterm infants: prevention is better than cure!," American Journal of Perinatology, vol. 25, no. 2, pp. 75-78, 2008.

[4] E. Tutdibi, K. Gries, M. Bücheler, B. Misselwitz, R. L. Schlosser, and L. Gortner, "Impact of labor on outcomes in transient tachypnea of the newborn: population-based study," Pediatrics, vol. 125, no. 3, pp. e577-e583, 2010.

[5] E. Hummler, P. Barker, F. Beermann et al., "Role of the epithelial sodium channel in lung liquid clearance," Chest, vol. 111, no. 6, p. 113S, 1997.

[6] N. L. Anderson, "The clinical plasma proteome: a survey of clinical assays for proteins in plasma and serum," Clinical Chemistry, vol. 56, no. 2, pp. 177-185, 2010.

[7] C. Kirchhoff, I. Habben, R. Ivell, and N. Krull, "A major human epididymis-specific cDNA encodes a protein with sequence homology to extracellular proteinase inhibitors," Biology of Reproduction, vol. 45, no. 2, pp. 350-357, 1991.

[8] I. Hellström, J. Raycraft, M. Hayden-Ledbetter et al., "The HE4 (WFDC2) protein is a biomarker for ovarian carcinoma," Cancer Research, vol. 63, no. 13, pp. 3695-3700, 2003.

[9] J. Wan, Y. Wang, G. Cai et al., "Elevated serum concentrations of HE4 as a novel biomarker of disease severity and renal fibrosis in kidney disease," Oncotarget, vol. 7, no. 42, pp. 6774867759, 2016. 
[10] B. Nagy Jr., B. Nagy, L. Fila et al., "Human epididymis protein 4: a novel serum inflammatory biomarker in cystic fibrosis," Chest, vol. 150, no. 3, pp. 661-672, 2016.

[11] Q. Zeng, M. Liu, N. Zhou, L. Liu, and X. Song, "Serum human epididymis protein 4 (HE4) may be a better tumor marker in early lung cancer," Clinica Chimica Acta, vol. 455, pp. 102106, 2016.

[12] L. Bingle, S. S. Cross, A. S. High et al., "WFDC2 (HE4): a potential role in the innate immunity of the oral cavity and respiratory tract and the development of adenocarcinomas of the lung," Respiratory Research, vol. 7, no. 1, article 452, 2006.

[13] L. Bingle, V. Singleton, and C. D. Bingle, "The putative ovarian tumor marker gene HE4 (WFDC2), is expressed in normal tissues and undergoes complex alternative splicing to yield multiple protein isoforms," Oncogene, vol. 21, no. 17, pp. 27682773, 2002.

[14] J. Li, H. Chen, A. Mariani et al., "HE4 (WFDC2) promotes tumor growth in endometrial cancer cell lines," International Journal of Molecular Sciences, vol. 14, no. 3, pp. 6026-6043, 2013.

[15] S. Yamashita, K. Tokuishi, T. Moroga et al., "Serum level of HE4 is closely associated with pulmonary adenocarcinoma progression," Tumor Biology, vol. 33, no. 6, pp. 2365-2370, 2012.

[16] K. Iwahori, H. Suzuki, Y. Kishi et al., "Serum HE4 as a diagnostic and prognostic marker for lung cancer," Tumor Biology, vol. 33, no. 4, pp. 1141-1149, 2012.

[17] E. Bignotti, M. Ragnoli, L. Zanotti et al., "Diagnostic and prognostic impact of serum HE4 detection in endometrial carcinoma patients," British Journal of Cancer, vol. 104, no. 9, pp. 1418-1425, 2011.

[18] A. Piek, W. C. Meijers, N. F. Schroten, R. T. Gansevoort, R. A. de Boer, and H. H. W. Silljé, "HE4 Serum Levels Are Associated with Heart Failure Severity in Patients With Chronic Heart Failure," Journal of Cardiac Failure, vol. 23, no. 1, pp. 12-19, 2017.

[19] C. D. Bingle and A. Vyakarnam, "Novel innate immune functions of the whey acidic protein family," Trends in Immunology, vol. 29, no. 9, pp. 444-453, 2008.

[20] P. Lahdenne, P. Kuusela, M. A. Siimes, K. A. Ronnholm, L. Salmenpera, and M. Heikinheimo, "Biphasic reduction and concanavalin a binding properties of serum alphafetoprotein in preterm and term infants," The Journal of Pediatrics, vol. 118, no. 2, pp. 272-276, 1991.

[21] S. L. Pauniaho, O. Tatti, P. Lahdenne et al., "Tumor markers AFP, CA 125, and CA 19-9 in the long-term follow-up of sacrococcygeal teratomas in infancy and childhood," Tumor Biology, vol. 31, no. 4, pp. 261-265, 2010.

[22] P. J. Lamy, C. Plassot, and J. L. Pujol, "Serum HE4: an independent prognostic factor in non-small cell lung cancer," PLoS One, vol. 10, no. 6, article e0128836, 2015.

[23] L. Hertlein, P. Stieber, A. Kirschenhofer et al., "Human epididymis protein 4 (HE4) in benign and malignant diseases," Clinical Chemistry and Laboratory Medicine, vol. 50, no. 12, pp. 2181-2188, 2012.

[24] D. J. Brennan, A. Hackethal, A. M. Metcalf et al., "Serum HE4 as a prognostic marker in endometrial cancer-a population based study," Gynecologic Oncology, vol. 132, no. 1, pp. 159$165,2014$.

[25] A. A. Ahmed and A. M. Abdou, "Diagnostic accuracy of CA125 and HE4 in ovarian carcinoma patients and the effect of confounders on their serum levels," Current Problems in Cancer, vol. 43, no. 5, pp. 450-460, 2019.
[26] M. T. Galgano, G. M. Hampton, and H. F. Frierson Jr., "Comprehensive analysis of HE4 expression in normal and malignant human tissues," Modern Pathology, vol. 19, no. 6, article BF3800612, pp. 847-853, 2006.

[27] S. Hammarstrom, "The carcinoembryonic antigen (CEA) family: structures, suggested functions and expression in normal and malignant tissues," Seminars in Cancer Biology, vol. 9, no. 2, pp. 67-81, 1999.

[28] C. Sauzay, A. Petit, A. M. Bourgeois et al., "Alpha-foetoprotein (AFP): a multi-purpose marker in hepatocellular carcinoma," Clinica Chimica Acta, vol. 463, pp. 39-44, 2016.

[29] Y. Otsubo, K. Hashimoto, T. Kanbe, M. Sumi, and H. Moriuchi, "Association of cord blood chemokines and other biomarkers with neonatal complications following intrauterine inflammation," PLoS One, vol. 12, no. 5, article e0175082, 2017. 